\title{
Spin-Dependent Resonant Tunneling in Semiconductor Nanostructures
}

\author{
Erasmo A. de Andrada e Silva \\ Instituto Nacional de Pesquisas Espaciais \\ CP515, 12201 São José dos Campos, São Paulo, Brasil \\ and Giuseppe C. La Rocca \\ Dipartimento di Fisica, Universitá di Salerno \\ 84081 Baronissi (Sa), Italia \\ and INFM, Scuola Normale Superiore, 56126 Pisa, Italia
}

Received February 6, 1999

\begin{abstract}
The spin-dependent quantum transport of electrons in non magnetic III- $\mathrm{V}$ semiconductor nanostructures is studied theoretically within the envelope function approximation and the Kane model for the bulk. It is shown that an unpolarized beam of conducting electrons can be strongly polarized in zero magnetic field by resonant tunneling across asymmetric double-barrier structures, as an effect of the spin-orbit interaction. The electron transmission probability is calculated as a function of energy and angle of incidence. Specific results for tunneling across lattice matched politype $G a_{0.47} \operatorname{In}_{0.53} A s / \operatorname{InP} / G a_{0.47} \operatorname{In}_{0.53} A s / G a A s_{0.5} S b_{0.5} / G a_{0.47} \operatorname{In}_{0.53}$ As double barrier heterostructures show sharp spin split resonances, corresponding to resonant tunneling through spin-orbit split quasi-bound electron states. The polarization of the transmitted beam is also calculated and is shown to be over $50 \%$.
\end{abstract}

The spin dependence of the electronic properties of artificial nanostructures is one of the leading problems in the physics of electronic devices. The interest lays both on the improvement of actual devices, as the GaAs polarized electron source (GaAs-PES) [1], and on the search for new devices [2]. The effects of the spin degree of freedom on the electron quantum confinement in III-V semiconductor nanostructures have been considered experimentally [3] as well as theoretically [4, 5, 6] with good agreement. On the other hand, while a better understanding of the spin-dependent electron transmission through ferromagnetic metal thin layers [7] and tunnel junctions [8] has been recently obtained, very little has been done to elucidate the microscopic mechanisms of electron spin polarized transport across nonmagnetic semiconductor heterostructures [9].

The spin dependence of the electronic properties of such structures in zero applied magnetic field originate from the spin-orbit interaction. The breaking of spin degeneracy in the conducting subbands when the system lacks inversion symmetry is of particular interest to electron both optical and transport properties [6]. Such symmetry in common GaAs heterostructures is in general broken by both microscopic and macroscopic contributions to the electron potential. They are due to the different atoms in the unit cell and to the asymmetries in the band gap engineering, respectively, and produce different wave vector or $\mathbf{k}$ dependence of the energy splitting between states with opposite spins. This in turn leads to spin-dependent electron transport effects of a somewhat different character.

In this paper we present the results of our investigations on the effects of the macroscopic specular asymmetry on the spin dependent electron quantum (coherent and vertical) transport in III- $V$ nanostructures [10]. Such effects come from the so-called Rashba spinorbit term, which, besides being adjustable according to the asymmetry fabricated, has been shown to be the strongest one in the case of structures with narrow gap materials $[4,6]$. As the main result, we obtain a new spin dependent resonant tunneling effect that can in principle be used for electron spin polarization. The effects of the $k^{3}$ term from the microscopic inversion 
asymmetry are going to be treated in a separated publication.

The Rashba spin-orbit term can be derived from general symmetry arguments [11].It does not depend on the structure orientation with respect to the crystal axis; depends only on the angle $\theta$ between the growth direction $(\hat{z})$ and the electron's wave-vector $\vec{k}$, in fact it can be written as

$$
H_{s o}=\frac{d}{d z} \beta(z, E)|\vec{k}| \sin \theta=\frac{d}{d z} \beta(z, E) k_{\|} .
$$

The coupling parameter $\beta$ as given by the eight band Kane model reads [6]

$$
\beta(z, E)=\frac{P^{2}}{2}\left(\frac{1}{E-U(z)-E_{v}(z)}-\frac{1}{E-U(z)-E_{v}(z)+\Delta(z)}\right),
$$

where $U(x)$ is the electrostatic potential from the depletion layer or applied external field, $E_{v}$ is the edge of the valence band, $\Delta$ is the spin orbit splitting in the valence band and $P$ is the interband momentum matrix element. Simple spin-dependent boundary conditions for the envelope functions can be derived in the presence of this term [6] and the problem of the spindependent quantum transport can be studied with the standard wave mechanics procedure.

Let's then consider the problem of an electron scattered by an ideal asymmetric double barrier potential with perfect translation symmetry along the plane of the interfaces, as illustrated in Figure 1. In the case of normal incidence $(\theta=0)$ the electron wave-vector has no component parallel to the interfaces, i.e. $k_{\|}=0$, there is no spin-orbit interaction and one has the usual spin independent resonant tunneling problem. If instead the crossing is oblique $(\theta \neq 0)$ the non zero electron's $k_{\|}$is conserved and the result for the transmission probability will depend on the orientation of the electron spin. Here we obtain such transmission probability from the solution of the stationary problem $H_{ \pm} F_{ \pm}=E F_{ \pm}$, for each spin orientation along $\hat{y}$. In the flat-band and zero-bias conditions, the envelope function in the layer material $j$ is of the form $F_{ \pm}=e^{i k_{\|} x}\left(A_{ \pm}^{j} e^{i k_{j} z}+B_{ \pm}^{j} e^{-i k_{j} z}\right)$, and the effective Hamiltonian is given by:

$$
H_{ \pm}(E)=-\frac{\hbar^{2}}{2} \frac{d}{d z} \frac{1}{m(E, z)} \frac{d}{d z}+E_{c}(z) \pm H_{s o}(E),
$$

where $E_{c}(z)$ gives the conduction band edge profile and

$$
\frac{1}{m(E, z)}=\frac{P^{2}}{\hbar^{2}}\left(\frac{2}{E-E_{v}(z)}+\frac{1}{E-E_{v}(z)-\Delta(z)}\right)
$$

is the inverse of the energy dependent effective mass as given by the same Kane model for the bulk.

Consider now an incoming electron with definite energy $E$ and spin $(+$ or -, i.e. up or down with respect to a direction perpendicular to both $\vec{k}_{\|}$and $\hat{z}$ ), and solve for the spin dependent transmission coefficient $t_{ \pm}$. Using standard transfer matrices the solution is straightforward:

$$
\left(\begin{array}{c}
t_{ \pm} \\
0
\end{array}\right)=M_{ \pm}^{2}\left(\begin{array}{cc}
e^{i k_{z} L} & 0 \\
0 & e^{-i k_{z} L}
\end{array}\right) M_{ \pm}^{1}\left(\begin{array}{c}
1 \\
r_{ \pm}
\end{array}\right)
$$

where the $M_{ \pm}^{j}, j=1,2$ are the spin-dependent transfer matrices corresponding to the two different barriers, $L$ is the well width and $k_{z}=\sqrt{\frac{2 m_{0}(E)}{\hbar} E} \cos (\theta)$ is the electron's wave-vector along the growth direction. The transfer matrix are obtained directly from the spin-dependent boundary conditions [6] and can be written as:

$$
M_{ \pm}^{j}=\frac{m_{0} m_{j}}{2 k_{z} \rho_{j}} \sinh \left(\rho_{j} d_{j}\right)\left(\begin{array}{cc}
e^{-i k_{z} d_{j}} & 0 \\
0 & e^{i k_{z} d_{j}}
\end{array}\right)\left(\begin{array}{cc}
P & Q_{ \pm} \\
Q_{ \pm}^{*} & P^{*}
\end{array}\right),
$$


with

$$
P=\frac{2 k_{z} \rho_{j}}{m_{0} m_{j}} \frac{1}{\tanh \left(\rho_{j} d_{j}\right)}+i\left[\left(\frac{2 k_{\|}}{\hbar}\right)^{2}\left(\beta_{0}-\beta_{j}\right)^{2}+\left(\frac{k_{z}^{2}}{m_{0}^{2}}-\frac{\rho_{j}^{2}}{m_{j}^{2}}\right)\right]
$$

and

$$
Q_{ \pm}=\frac{4 k_{z} k_{\|}}{\hbar^{2} m_{0}}\left(\beta_{0}-\beta_{j}\right)+i\left[\left(\frac{2 k_{\|}}{\hbar}\right)^{2}\left(\beta_{0}-\beta_{j}\right)^{2}-\left(\frac{k_{z}^{2}}{m_{0}^{2}}+\frac{\rho_{j}^{2}}{m_{j}^{2}}\right)\right],
$$

where $d_{j}$ is the $j$ barrier width, $\hbar k_{\|}=\sqrt{2 m_{0} E} \sin \theta$ is the conserved momentum parallel to the interfaces and $\rho_{j}=\sqrt{\frac{2 m_{i}}{\hbar^{2}}\left(E_{c}^{j}-E\right)+k_{\|}}$is the decay coefficient of the evanescent wave inside the barrier. We remember that $\left\{m_{0}, \beta_{0}\right\}$ and $\left\{m_{j}, \beta_{j}\right\}$, the well and barrier material parameters respectively, are energy dependent in accord to the expressions above. In the limit of $\beta_{0}=\beta_{j}=0$ (or $\Delta_{0}=\Delta_{j}=0$ ), i.e. no spin orbit interaction, the above transfer matrix reduces to the usual spin-independent expression [12].

If one now consider an unpolarized beam of conducting electrons it is possible to calculate the polarization of the transmitted beam defined by:

$$
P(E, \theta)=\frac{T_{+}(E, \theta)-T_{-}(E, \theta)}{T_{+}(E, \theta)+T_{-}(E, \theta)}
$$

where $T_{ \pm}=t_{ \pm} t_{ \pm}^{*}$ is the spin dependent transmission probability. The above obtained transfer matrix can be used also to calculate the spin dependent tunneling across single asymmetric barries, but the polarization of the transmitted beam one obtains is quite small [9]. A much larger polarization can be obtained with double barriers, as shown bellow.

As a practical and realistic example we have calculated the transmission probability and the polarization of the transmitted beam for a lattice matched politype $G a_{0.47} I n_{0.53} \mathrm{As} /$ InP/Ga $a_{0.47}$ In $_{.53} A s / G a A s_{0.5} S b_{0.5} / G a_{0.47} I_{0.53} A s$ double barrier structure. As shown in Figure 2 we obtain sizable spin splittings in the sharp resonances. A splitting of a few $\mathrm{meV}$ is for instance observed between the transmission peaks connected with the resonant tunneling through the second quasi-bound subband. The effect is much smaller for tunneling through the ground subband in accord to the smaller spin-orbit splitting [6]. The corresponding polarization of the transmitted beam has been plotted in the lower part. The obtained polarization at resonance is in this case over $50 \%$. Even larger polarizations can be obtained not only with the use of new material combinations, but also with a careful optimization of the asymmetric structure parameters $d_{1}, d_{2}$ and $L$. We have shown here the results only as a function of energy for a fixed $\theta$, but it is clear that the results are similar as a function of $\theta$ for a fixed energy.

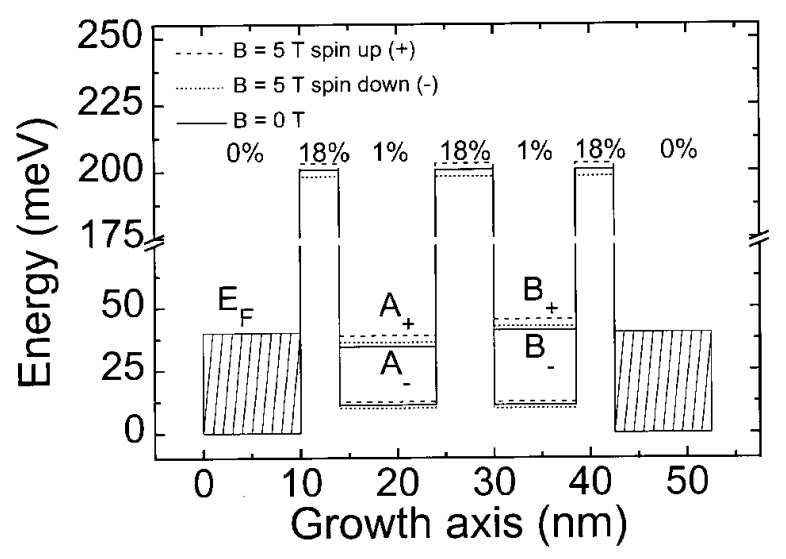

Figure 1. Illustration of the spin polarized electron quantum transport problem discussed in the paper: resonant tunneling through asymmetric double barrier structures.

Summarizing, we have presented a study of the electron spin polarization effects due to the Rashba spinorbit term in the resonant tunneling through asymmetric double barrier structures. Specific calculations were performed for the spin-dependent electron transmission probability across politype double barrier structures, showing spin-split resonances separated by a few $\mathrm{meV}$ and able to polarize the transmitted beam up to over $50 \%$. The continuous improvement in the nanolithography technology allows us to believe that in a near future it will be possible to test and apply the present theory. Samples in which electrons are injected in and collected from double barrier semiconductor structures with a preferable angle with respect to the growth direction could for example be fabricated. 


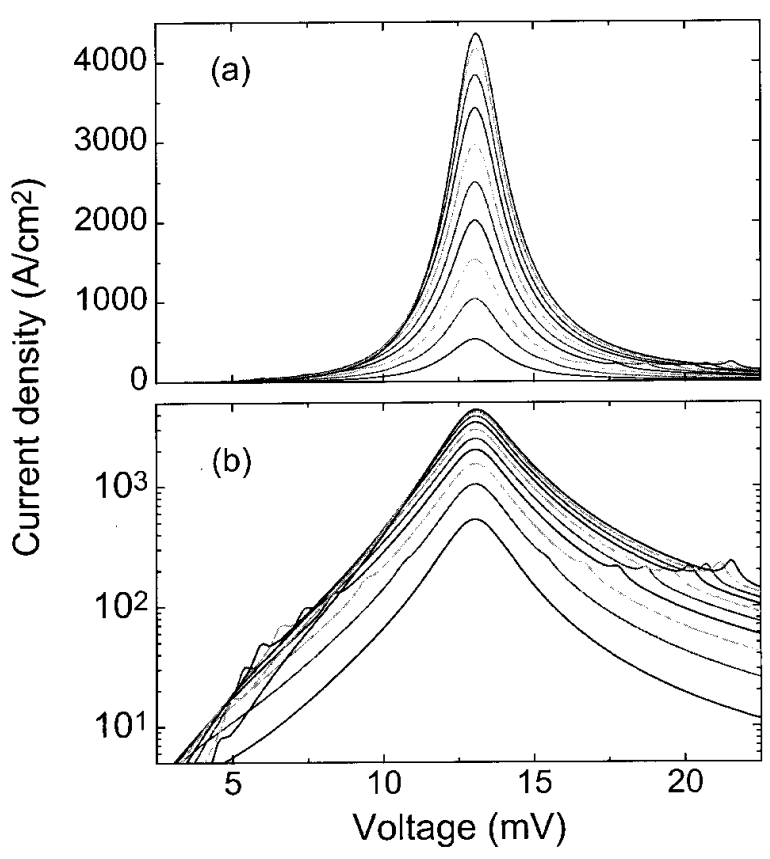

Figure 2. Obtained spin-dependent electron transmission probability for crossing an asymmetric double barrier $G a_{0.47} \operatorname{In}_{0.53} A s / \operatorname{In} P / G a_{0.47} \operatorname{In}_{0.53} A s / G a A s_{0.5} S b_{0.5} /$ $G a_{0.47} \operatorname{In}_{0.53}$ As politype lattice matched structure, illustrated in Figure 1. The two doublets correspond to transmission through the ground and first excited spin-split quasi-bound states respectively. In the lower panel is plotted the polarization of the transmitted beam. The bulk parameters used are the low temperature values reported in the Landolt-Börstain Tables. We used $L=150 \AA{ }^{\circ}, \theta=\frac{\pi}{4}$ and, for the $\operatorname{In} P$ and $G a A s .5 S b .5$ barriers, $d_{1}=40 \AA$, $V_{1}=0.4 \mathrm{eV}$ and $d_{2}=40 \AA V_{2}=.15 \mathrm{eV}$ respectively.

This work was supported by FAPESP (96/00058-1) and CNPq (300047/91-6), Brasil.

\section{References}

[1] See for instance,D.T. Pierce and R.J. Celotta in "Optical Orientation", F. Meier and B.P. Zakharchenya, eds.; Modern Problems in Condensed Matter Sciences Vol. 8 (Elsevier Sciences Publishers, 1984).

[2] See, for example, special issue of Physics Today, April 1995.

[3] J. Luo et al, Phys. Rev. B 41, 7685 (1990); B. Das et al, Phys. Rev. B 39, 1411 (1989); B. Jusserand et al., Phys. Rev. B 51, 4707 (1995), G. Engeles et al., Phys. Rev. B 55, R1958 (1997).

[4] P.V. Santos e M. Cardona, Phys. Rev. Lett. 72, 432 (1994); R. Winkler and U. Rossler Phys. Rev. B 48 8918 (1993) , P. Pfeffer Phys. Rev. B 55, R7359 (1997).

[5] E. A. de Andrada e Silva, Phys. Rev. B 46, 1921 (1992)

[6] E. A. de Andrada e Silva, G. C. La Rocca and F. Bassani, Phys. Rev. B 50, 8523 (1994) and 55, 16293 (1997).

[7] A. Filipe et al., Phys. Rev. Lett. 80, 2425 (1998).

[8] S.T. Chui, Phys. Rev. B. 55, 5600 (1997).

[9] see, for instance, A. Voskoboynikov, S. Shin Liu and C.P. Lee, Phys. Rev. B 58, 15397 (1998).

[10] see, for a preliminary publication of the results, E. A. de Andrada e Silva and G. C. La Rocca, Phys. Rev. B 59, R15583 (1999).

[11] Yu. A. Bychkov and E. I. Rashba, J. Phys. C 17, 6039 (1984).

[12] see, for example, "Quantum Phenomena", S. Datta (Addison-Wesley Modular Series on Slod State Devices, Vol. VIII, 1989). 